\title{
Polysèmes
}

Revue d'études intertextuelles et intermédiales

\section{Recording Britain - John Piper et le patrimoine architectural anglais dans les années 1940}

Sophie Aymes

\section{OpenEdition}

\section{Journals}

Édition électronique

URL : http://journals.openedition.org/polysemes/628

DOI : $10.4000 /$ polysemes.628

ISSN : 2496-4212

Éditeur

SAIT

Édition imprimée

Date de publication : 1 janvier 2008

Pagination : 59-72

ISSN : 0999-4203

\section{Référence électronique}

Sophie Aymes, « Recording Britain - John Piper et le patrimoine architectural anglais dans les années 1940 », Polysèmes [En ligne], 10 | 2008, mis en ligne le 01 mars 2015, consulté le 06 mai 2019. URL http://journals.openedition.org/polysemes/628 ; DOI : 10.4000/polysemes.628

Ce document a été généré automatiquement le 6 mai 2019.

Polysèmes 


\title{
Recording Britain - John Piper et le patrimoine architectural anglais dans les années 1940
}

\author{
Sophie Aymes
}

1 Pendant la Seconde Guerre Mondiale, l'artiste John Piper (1903-1992) parcourt l'Angleterre pour rendre compte des destructions causées par les bombardements et pour peindre les bâtiments encore intacts, lors d'une véritable course contre la montre. Il renoue avec ses parcours obsessionnels de la campagne anglaise pendant l'adolescence. Son itinéraire individuel coïncide avec le mouvement national de sauvegarde patrimoniale qui s'inscrit dans l'effort de guerre britannique, et avec la volonté de nombreux artistes et historiens de l'art de redéfinir un art national en effectuant un retour aux sources.

2 Recording Britain est le titre de l'un des programmes de sauvegarde patrimoniale auxquels John Piper participe. Ce titre pourrait aussi définir son entreprise artistique. En effet, ses parcours le conduisent sur les pas des grands paysagistes, aquarellistes, topographes et « touristes » en quête de pittoresque, tandis qu'il revendique l'héritage de William Blake et de Samuel Palmer. Piper visite les sites qu'ils ont peints ou dépeints, et revisite leurs textes. Quelques indications biographiques préalables permettront de voir que l'attitude artistique de John Piper se construit au gré de ses parcours, au sens littéral du terme. Je m'attacherai ensuite à définir la nature paradoxale de son entreprise artistique, liée à sa vision de la guerre et de la reconstruction. L'attrait que les ruines exercent sur l'artiste permettra plus particulièrement de s'interroger sur le contraste entre déliquescence architecturale et accrétion intertextuelle.

\section{Parcours artistique ${ }^{1}$}

3 John Piper est né en 1903, dans une famille aisée de la middle-class du Surrey. Il mourra en 1992. Dès l'adolescence, il parcourt de manière obsessionnelle la campagne et développe une prédilection particulière pour les églises et leurs vitraux. Il collectionne également 
les guides touristiques, notamment les ouvrages illustrés par Frederick Landseer Griggs. Sa formation artistique commence sur le tard après la mort de son père en 1927 et ne dure que trois ans : après être passé par la Richmond School of Art, il étudie deux ans au Royal College of Art. Il intègre la jeune scène artistique londonienne, mais s'intéresse aussi bien aux paysagistes, topographes et peintres d'architecture anglais des XVIII ${ }^{\mathrm{e}}$ et $\mathrm{XIX}^{\mathrm{e}}$ siècles qu'aux avant-gardes continentales, et travaille déjà sur toutes sortes de supports. Vers 1929, il se lance dans la critique d'art pour divers magazines, mais surtout, à partir de 1936, il commence à collaborer régulièrement à l'Architectural Review, qui est une source essentielle pour comprendre les enjeux de la reconstruction.

4 Piper se lance dans la peinture abstraite à partir de 1935, période de formation qui lui permet d'acquérir une discipline picturale en termes de couleur et de texture. Il exécutait déjà des collages et des constructions abstraites depuis 1932. Puis il se détourne brusquement de l'abstraction et revient à la peinture figurative et au paysage anglais. $\mathrm{Ce}$ tournant est annoncé et justifié dans l'article "England's Climate ", écrit avec le poète Geoffrey Grigson et publié dans le $n^{\circ} 7$ de la revue Axis (1936), l'organe des artistes abstraits anglais, dont la rédactrice en chef est sa future seconde épouse Myfanwy Evans : " we believed in an opposition of life to art. But our job is to feel history, to feel art and life. We must join history [...] $»^{2}$. Le retour à la figuration est lié à la volonté de définir le rôle de l'artiste dans la société, et, rapidement, son rôle en temps de guerre, selon une vision de la fonction morale de l'art aux antipodes de la conception esthétique de Roger Fry $^{3}$. Dès 1938, Piper est certain que la guerre va éclater, et songe déjà à la reconstruction de l'après-guerre dans le domaine de l'art.

5 À cette époque, le rédacteur en chef de l'Architectural Review, J.M. Richards, lui présente John Betjeman, qui avait occupé ce poste jusqu'en 1936. John Betjeman propose à Piper de collaborer à la série des Shell Guides ${ }^{4}$. Piper a alors repris sa collection de guides touristiques anglais, avec une prédilection pour les ouvrages du XVIII ${ }^{e}$ siècle et du début du XIX ${ }^{e}$ siècle, influencés par les théories esthétiques de William Gilpin ${ }^{5}$ et de Uvedale Price ${ }^{6}$, qui codifient et vulgarisent alors l'esthétique pittoresque. Piper commence aussi à peindre les grandes country houses d'Angleterre et du pays de Galles. Sa première commande émane de Duckworth Press en 1940: il exécute une série de peintures de Stowe (Buckinghamshire), où William Gilpin situe son Dialogue (1757) et qui lui fournit un cadre visuel de première importance ${ }^{7}$. Viennent aussi les commandes de ceux qui seront ses mécènes: en 1941, Sir Osbert Sitwell lui demande de peindre Renishaw Hall, la demeure familiale, pour illustrer son autobiographie ${ }^{8}$ [illustration 1]. En 1943, Edward Sackville-West lui demande de peindre Knole [illustration 2]. Knole étant la demeure d'Orlando, on peut voir dans cette commande une transition entre deux époques, deux cercles de mécènes et de groupes artistiques. En réaction contre le formalisme et le cosmopolitisme incarnés par Roger Fry et le Bloomsbury group se développe depuis le milieu des années 1930 un nouveau courant critique et historiographique auquel participent les historiens de l'art Christopher Hussey et Nikolaus Pevsner, l'artiste Michael Ayrton ou encore le directeur de la National Gallery, Kenneth Clark, qui fréquente le salon de Sir Osbert Sitwell et joue un rôle crucial dans la politique culturelle britannique.

6 Après la déclaration de guerre britannique et l'épisode de la «drôle de guerre », la Bataille d'Angleterre s'engage en août 1940. Piper va participer à plusieurs programmes de préservation patrimoniale. Kenneth Clark le recrute comme artiste de guerre au sein du WAAC (War Artists' Advisory Committee, fondé en 1940) qu'il préside avec Lord 
Macmillan, et qui va institutionnaliser l'art de guerre. C'est alors que John Piper se met à parcourir les sites bombardés pour rendre compte des destructions. La réception de son œuvre est donc liée à la guerre, et il rencontre un succès progressif, grâce à des toiles telles que Coventry Cathedral, 15 November 1940 et House of Commons 1941: Aye Chamber [illustrations $3 \& 4$ ]. Lorsque débutent les Baedecker raids ${ }^{9}$, il est envoyé à Bath où il trouve le sujet de l'une de ses toiles les plus célèbres, Somerset Place, Bath (1942) [illustration 5].

D'autre part, en août 1941, sur la recommandation de Kenneth Clark, il reçoit une commande royale. George VI souhaite qu'il exécute des aquarelles de Windsor Castle en prenant pour modèle les aquarelles topographiques que Paul Sandby réalisa pour George III dans les années 1760. Enfin, une autre campagne patrimoniale est lancée en 1940, par le Pilgrim Trust sous l'égide du Ministry of Labour and National Service. Elle s'intitule Recording Britain. L'entreprise est dirigée par Kenneth Clark sur les conseils de Paul Nash qui lui fournit également les listes d'artistes à employer au sein du WAAC. Quatre-vingt-dix-sept artistes vont participer et produire plus de 1500 dessins et aquarelles topographiques reproduits dans 4 volumes publiés de 1946 à 1949. John Piper est chargé de trois comtés et il s'arrange pour voir les aquarelles de John Sell Cotman dans la collection de Sir Michael Sadler.

Ces commandes lui permettent d'approfondir ses recherches sur l'art anglais et il publie en 1942, l'essai British Romantic Artists dans lequel il réécrit l'histoire de l'art anglais à partir des années 1750 selon l'idée d'une filiation romantique qui a parfois connu des éclipses et dont il se fait l'héritier. Il rejoint alors le mouvement de redécouverte des œuvres de William Blake et Samuel Palmer. Il entame d'ailleurs la rédaction de British Romantic Artists au moment où se tient l'exposition Turner de 1939, et avant que les collections des musées ne soient mises à l'abri. Ses recherches pour British Romantic Artists contribuent à son itinéraire personnel de redécouverte des artistes incarnant désormais le génie anglais. Il veut voir ce qu'ils ont vu, comme ils l'ont vu. Il renoue avec la convention de l'esthétique pittoresque qui consiste à lire le paysage au travers d'un prisme : le touriste éclairé voyait le paysage au travers d'un écran de références textuelles et picturales. Dès 1937, il entame des painting tours dans des endroits isolés, au pays de Galles (déjà parcouru par Samuel Palmer, Edward Calvert ou William Turner) et dans le Yorkshire (notamment à Gordale Scar, déjà (dé)peint par William Turner, Thomas Gray ou encore Thomas Girtin). Les lieux peints par les artistes « romantiques » méritent donc le détour. Ses propres travaux dénotent un intérêt égal pour la pierre construite et pour la pierre naturelle. A l'instar de William Gilpin, dont il dit: «[he] covered the ground of England $»^{10}$, ces artistes ont signalisé le paysage et influencé plusieurs générations d'auteurs de guides touristiques, tels que les guides Murray ou les guides Baedecker, justement.

\section{Ambivalence de la reconstruction}

En juillet 1941, dans le numéro spécial intitulé «Destruction and Reconstruction », l' Architectural Review change de politique éditoriale devant l'ampleur des destructions causées par les bombardements ${ }^{11}$. Désormais chaque numéro comportera un supplément constitué de photographies des bâtiments bombardés et de notices (" obituary records » ${ }^{12}$ ), et envisagera les modalités de la reconstruction. Or, ces modalités sont très ambivalentes. La guerre n'est décrite qu'au travers des destructions architecturales (en majeure partie, il s'agit d'architecture monumentale, religieuse ou politique), 
destructions que l'on déplore, mais qui sont également considérées comme une chance historique. C'est l'occasion de mettre enfin en œuvre une véritable planification urbaine résolument tournée vers le futur et tenant compte des avancées technologiques dans l'industrie du bâtiment (présentées au travers des nombreuses pages publicitaires de chaque numéro) ${ }^{13}$. Cette période est parfois aussi décrite comme un nouveau départ offrant la possibilité de mettre un frein au développement urbain et suburbain anarchique de l'entre-deux-guerres. De manière générale, les articles de l'Architectural Review illustrent l'ambiguïté du discours politique sur la reconstruction, manifeste dans l'épisode de la reconstruction de la cathédrale de Coventry ${ }^{14}$.

Pour Piper, l'idée de ce nouveau départ est fondée sur la référence à la période dite « romantique » qui jouera le rôle de genèse. Les artistes romantiques deviennent des précurseurs mythiques, et leurs œuvres, des hypo-icônes et hypotextes fondateurs dont la contemplation peut revitaliser l'art contemporain. Ce détour permet de contourner l'entre-deux-guerres et ses avant-gardes européennes continentales, ainsi que l'ère victorienne, périodes assimilées à l'expansion industrielle britannique et, en filigrane, à la montée des classes moyennes et populaires qui est mentionnée de manière indirecte dans certains articles de l'Architectural Review lorsque l'on déplore le développement du tourisme de masse ${ }^{15}$. John Piper est certes un touriste, mais il veut appartenir à la classe des gentilshommes éclairés partant pour un Grand Tour, en quête de pittoresque, armés d'un Claude glass (dont l'avatar moderne est, dans son cas, l'appareil photo), d'un carnet de croquis et d'un guide rempli d'injonctions quant à la manière de voir le paysage. Piper renouvelle cette expérience médiatisée du paysage qui se veut réelle et authentique. Il a également une place privilégiée auprès de grands propriétaires terriens qui lui passent commande et le félicitent pour ses vues.

11 Dans son article de 1945 sur la country house Seaton Delaval (Northumberland) construite par le dramaturge architecte Sir John Vanbrugh, Piper explique que deux incendies (en 1752 et 1822) lui ont donné une apparence contemporaine : « extremely up-to-date: very much of our times $»^{16}$ [illustration 6]. La couleur de feu de sa façade, symbole de destruction et d'apothéose, est due aux incendies mais aussi à l'effet climatique du vent d'est («ochre and flame-licked red, pockmarked and stained in purplish umber and black », 43). Il écrit : " House and landscape are seared by the east wind that blows from Germany, and riven with fretting industrialism » (43). Or le vent d'est ravageur qui souffle d'Allemagne vient aussi du continent (et surtout de Paris) où règnent les avant-gardes artistiques. De plus, les dommages causés par la guerre sont implicitement comparés aux changements provoqués aussi bien par la révolution industrielle que par les organismes de conservation patrimoniale comme le National Trust ${ }^{17}$. Dans cette géographie symbolique, les régions côtières sont particulièrement sensibles et lorsque Piper abandonne l'abstraction, il prône dans l'article «Abstraction on the Beach" (1938) un " art de la reconstruction » (" art of reconstruction ») $)^{18}$. Il faut tout reprendre à zéro en partant de la plage. Le bord de mer, où il réalise des collages, est source d'inspiration. La ligne de côte et la Manche sont des espaces de transition entre Paris et Londres, mais aussi le lieu d'où vient le danger ${ }^{19}$.

12 De même, le programme Recording Britain met l'accent sur les comtés du sud-est de l'Angleterre, les zones les plus exposées. Comme l'indique le sous-titre « Record(ing) the changing face of England ", il s'agit de témoigner des changements qui surviennent en Angleterre et de recenser les éléments menacés ${ }^{20}$. L'introduction du premier volume montre que le mot «record» est aussi ambivalent que l'image du vent chez Piper : on 
invite les artistes à peindre les lieux et bâtiments présentant un intérêt national, et en particulier ceux exposés aux destructions de la guerre, mais on souligne aussi qu'en dépit du travail de conservation du National Trust et autres organismes, l'aspect extérieur de la Grande-Bretagne subissait déjà des changements bien trop rapides avant la guerre, à cause de l'action des rénovateurs et spoliateurs ${ }^{21}$.

Dans le recueil Landscape and Power publié sous la direction de W.J.T. Mitchell, Ann Bermingham analyse les fondements idéologiques du paysage en tant que discours politique et artistique de la fin du XVIII ${ }^{e}$ siècle. Le radicalisme français est alors vu en Angleterre comme " une menace contre l'ordre naturel de la société $»^{22}$, dans la mesure où les révolutionnaires prétendent gouverner le peuple selon des principes abstraits, en total désaccord avec la conception qu'ont les Whigs et Tories anti-jacobéens de la nature humaine et de l'évolution naturelle de la société (symbolisée par le développement organique de la constitution anglaise). Ann Bermingham résume son analyse par une série d'oppositions entre système abstrait, conceptuel, synthétique (où se rangent les principes constitutionnels révolutionnaires) et système analytique, réaliste, naturaliste, reposant sur un rejet de l'abstraction et sur la valorisation du trait individuel et de la variété, et représentant le fondement idéologique d'une vision conservatrice de la hiérarchie sociale comme état naturel (auquel appartient le rêve utopique d'un langage pictural universel, miroir stable de son référent naturel).

Or, on constate que les articles de l'Architectural Review consacrés à la reconstruction révèlent une tension entre un désir de planification moderne et démocratique, et un désir de conserver les bâtiments dans leur état naturel et individuel. Les numéros de guerre de la revue participent à la reconstruction idéologique d'une mythologie nationale et témoignent d'un recours privilégié au trope architectural pour symboliser la nation et son devenir ${ }^{23}$. Il est significatif de trouver à côté des articles prônant une reconstruction planifiée des textes servant de contrepoint et traitant de la reconstruction de Londres après l'incendie de 1666 lorsque s'opposaient les tenants de la conservation du tracé des rues de la City et ceux qui, comme Sir Christopher Wren, souhaitaient ouvrir de grandes artères, ou encore de la reconstruction haussmannienne de Paris et de la genèse du pittoresque. John Piper propose quant à lui de conserver les ruines résultant des bombardements ${ }^{24}$. Cette position surprenante est moralement intenable puisqu'elle ne tient pas compte du sort des victimes. On ne trouve d'ailleurs aucune photo de victime humaine des bombardements pour accompagner les «notices nécrologiques» ("obituaries») de l'Architectural Review. Les auteurs se positionnent d'emblée dans la sphère esthétique, et non dans la sphère morale. Ce choix est justifié par J.M. Richards dans l'introduction à la compilation de photographies extraites de la revue, The Bombed Buildings of Britain ${ }^{25}$. Les tableaux de John Piper ne contiennent pas de représentation humaine non plus. Ils sont cependant célébrés pour leur capacité à rendre l'atmosphère des lieux («mood»). Le terme " mood», très usité dans la critique artistique et littéraire de l'époque, désigne la relation esthétique entre le spectateur (qui, lui, n'est pas mis en scène en tant que sujet de l'œuvre) et ce qu'il regarde, ou plutôt une projection du plaisir esthétique que provoque la contemplation d'un objet alors jugé digne d'être contemplé.

L'ambivalence qui marque le discours de la reconstruction témoigne des visions conflictuelles du modèle de société à construire après la guerre. On la retrouve dans la pratique picturale et les écrits de John Piper. 


\section{«Pleasing decay »}

16 John Piper insiste constamment sur la nécessité de rééduquer le regard de ses contemporains pour leur réapprendre à voir ce qui mérite d'être vu, ce qu'il a hérité de l'artiste John Sell Cotman et qui est salué par ses admirateurs. Or, ce que Piper célèbre chez Cotman, c'est son goût du détail particulier, sa capacité à rendre l'aspect caractéristique de tel site dans toute son individualité, ce qui, selon lui, définit l'art romantique ${ }^{26}$. Son ouvrage British Romantic Artists débute par la phrase suivante: "Romantic art deals with the particular ${ }^{27}$, en écho aux célèbres annotations de William Blake sur son édition des œuvres de Joshua Reynolds.

17 L'architecture digne d'être recensée, c'est celle qui est affectée par ce que Piper appelle le "pleasing decay» ou "decrepit glory», oxymores forgés d'après l'expression " parasitical sublimity » tirée de The Seven Lamps of Architecture de John Ruskin (1849), et qui résument sa conception de l'esthétique pittoresque ${ }^{28}$. C'est pourquoi les églises des Fens étaient si belles à l'époque où John Sell Cotman les a dessinées: "Most country churches were at an extreme of beauty. Ready to drop like over-ripe fruit, they were in an exquisite state of decay $»^{29}$. Les ruines provoquées par les bombardements semblent résulter d'une accélération dramatique de ce processus de dégradation naturelle et ont la même valeur esthétique parce qu'elle rendent visibles les effets de l'histoire et symbolisent la continuité entre tradition et modernitée ${ }^{30}$.

18 Piper représente le «pleasing decay » dans ses textes et ses peintures. Ses articles sont des descriptions de parcours sur des chemins déjà parcourus, « couverts » (« covered »), vers des sites qui sont déjà des paysages, c'est-à-dire des lieux déjà recouverts d'un voile (d'une couche, pourrait-on dire) idéologique. Ces textes offrent en général la vue éloignée ou panoramique d'un bâtiment, puis une série de vues rapprochées aboutissant à une description de la pierre en gros plan. Au cours de ce rapprochement, le bâtiment subit une métamorphose métaphorique et se cristallise en roc. Sous l'effet du "pleasing decay ", le bâtiment retourne à la nature. Piper est fasciné par le " decay », crépi naturel à opposer au crépi des restaurateurs, à ce qu'il nomme « rot " ${ }^{31}$. Cette lèpre s'oppose à la pourriture noble du « decay » qui apparait sous de multiples formes, dont celle du lichen, à mi-chemin entre le règne végétal et le règne minéral. Son art du palimpseste se développe par accrétion intertextuelle. Ses descriptions reposent sur un effet de liste renforcé par les couches successives que forment les nombreuses citations. Virtuellement infinies, ces descriptions visent à nommer le moindre brin d'herbe, reproduire linguistiquement la variété du vivant, et tiennent de la logique du signe naturel.

19 Sur ses toiles, la couche de peinture recouvre presque le trait de crayon qui individualise la structure architecturale. Piper travaille la surface de ses toiles en les grattant, en traçant un réseau de signes avec le manche du pinceau. Ces ratures annulent la profondeur et couvrent la toile comme le "decay», la pierre. Cette technique picturale permet de signifier le « decay » à l'œuvre sur la surface des toiles et des murs, et renvoie à la relation esthétique qui fonde le choix de l'architecture comme sujet, relation que l'on peut résumer par un jeu de mot (approximatif) entre «mould» et «mood». Or la récurrence de ces signes picturaux, la composition frontale des toiles qui représentent des façades dont l'expression la plus aboutie est celle des country houses, l'utilisation des couleurs et des formes de manière quasi-abstraite forment un langage formel uniforme (et répétitif) qui fonde le style immédiatement reconnaissable de Piper. En cela, cette 
sémiotique visuelle est de nature synthétique, tandis que sa sémiotique textuelle est de nature analytique. Les deux tendances de son art (vers l'abstraction et le naturalisme) démontrent qu'il tente de concilier un idéal patricien d'un autre temps ${ }^{32}$ et les leçons incontournables de l'art moderne.

Peut-être peut-on considérer que cette tension trouve sa résolution dans son art du vitrail, où la vitrification met un terme final à la minéralisation de l'architecture. Pour lui, la patine du vitrail est l'équivalent du « decay », et donc symbole de continuité ${ }^{33}$. L'herbe poussant au pied des églises des Fens et décrite dans ses articles trouve aussi son équivalent vitrifié dans le reflet des vitraux sur le sol de ces églises, puis dans les éclats de verre jonchant le sol des bâtiments bombardés ${ }^{34}$. On peut ainsi voir dans le vitrail créé par Piper (1957-1961) pour le baptistère de la nouvelle cathédrale de Coventry l'aboutissement logique d'un parcours entamé dans les années 1930. De même, les décors qu'il dessine pour les opéras de Benjamin Britten après la guerre héritent de la théâtralité de ses toiles.

\section{Conclusion}

21 À partir de la deuxième moitié des années 1930, les artistes que l'on a appelés Néoromantiques élaborent leur vision pastorale d'une Arcadie anglaise. À la faveur de l'isolement britannique pendant la guerre, ils participent à la redéfinition d'un art national fondé sur une vision nostalgique et parfois élitiste de la culture anglaise héritée $\mathrm{du}$ romantisme. L'architecture est plus que jamais le symbole des valeurs qu'il faut préserver et de la continuation naturelle d'une société naturellement pérenne, ou du moins de sa vision pastorale mythique. Mais c'est le mode élégiaque de la pastorale qui l'emporte lorsque le paysage et le patrimoine architectural sont menacés, comme l'indique la devise « et in Arcadia ego » dans Brideshead Revisited. La country house est une expression des plus significatives du recours au trope architectural. En 1945 Sir Osbert Sitwell célèbre les œuvres de John Piper en ces termes : « at the very moment when the great English houses, the chief architectural expression of their country, are passing [...] a painter has appeared to hand them on to future ages $»^{35}$. Castle Howard (où l'adaptation télévisée de Brideshead Revisited fut tournée) et Seaton Delaval deviennent pendant la guerre des citadelles assiégées, pour reprendre la métaphore martiale utilisée par leur constructeur, l'architecte et dramaturge Vanbrugh ${ }^{36}$. Ces demeures sont en effet occupées par des bataillons de soldats et d'écoliers, autant de philistins qui dénaturent les lieux de manière aussi destructive que les bombardements ${ }^{37}$. C'est aussi pendant ces années de guerre que l'écrivain Mervyn Peake crée Gormenghast, demeure déliquescente - rongée elle aussi par le "decay» - des Comtes de Groan, famille dégénérescente qui laisse Steerpike, un jeune parvenu, se saisir du pouvoir. Chez Piper, le flamboiement de la façade de Seaton Delaval signe le déclin de la country house et d'une certaine esthétique. À la fin de la guerre, les fruits pourrissants du jardin pastoral sont près de se détacher et de tomber. 


\section{BIBLIOGRAPHIE}

\section{Euvres générales :}

Architectural Review 90 (juillet 1941).

Architectural Review 92 (juillet 1942), numéro spécial “The End of Reconstruction”.

Bermingham, A. "System, Order and Abstraction: The Politics of English Landscape Drawing Around 1795”. Landscape and Power. W.J.T. Mitchell (ed). Chicago: Chicago UP, (1994) 2002, 77-101.

Campbell, L. Coventry Cathedral. Art and Architecture in Post-War Britain. Oxford: Clarendon Press, 1996.

Fry, R. Vision and Design. London: Chatto \& Windus, 1929.

Hussey, C. “The Twilight of the Great House”. Country Life (January 1945): 153.

Ingrams, R. Piper's Places. London: Chatto \& Windus, 1983.

Jenkins, F.D. John Piper: The Forties. London: Philip Wilson Publishers \& Imperial War Museum, 2000 .

Jenkins, F.D. \& F. Spalding. John Piper in the 1930s. Abstraction on the Beach. London: Merrell, 2003.

Levinson, O. "Quality and Experiment": the Prints of John Piper: a Catalogue Raisonné, 1923-91. London: Lund Humphries, 1996.

McCormick, F. Sir John Vanbrugh. The Playwright as Architect. University Park: Pennsylvania State UP, 1991.

Mellor, D. (ed.). A Paradise Lost: The Neo-Romantic Imagination in Britain, 1935-1955. London: Lund Humphries \& The Barbican Art Gallery, 1987.

Osborne, J. John Piper and the Stained Glass. Stroud: Sutton, 1997.

Palmer, A. (ed.). Recording Britain. Oxford : OUP \& The Pilgrim Trust, 1946-49 (4 volumes).

Peake, M. Titus Groan. London: Eyre \& Spottiswoode, 1946.

Peake, M. Gormenghast. London: Eyre \& Spottiswoode, 1950.

Richards, J.M. The Bombed Buildings of Britain: a Record of Architectural Casualties. London: The Architectural Press, 1942.

Robinson, J.M. The Country House at War. London: Bodley Head, 1989.

Strong, R., M. Binney \& J. Harris (ed.). The Destruction of the Country House, 1875-1975. London: Thames \& Hudson, 1974.

Waterson, M. (ed.). The Country House Remembered: Recollections of Life Between the Wars. London: Routledge \& Kegan Paul, 1985.

Waugh, E. Brideshead Revisited. London: Chapman \& Hall, 1945.

Yorke, M. The Spirit of Place: Nine Neo-Romantic Artist and Their Times. London: Constable, 1988.

\section{Textes de John Piper :}


“Art or Anti-Art?” in Osborne, June. John Piper and the Stained Glass, 1997: 155-169.

“Abstraction on the Beach”. XX $X^{e}$ siècle, $1^{\text {er }}$ juillet 1938.

“The Architecture of Destruction”. Architectural Review 90 (juillet 1941): 25-26.

British Romantic Artists. London: William Collins, série Britain in Pictures, 1942.

Buildings and Prospects. London: The Architectural Press, 1948.

“England's Climate”. Axis 7 (automne 1936): 4-9. Article écrit avec Geoffrey Grigson.

John Piper's Stowe. Westerham: Hurtwood Press \& the Tate Gallery, 1983.

“John Sell Cotman". Architectural Review 92 (juillet 1942): 9-12.

“Picturesque Travel Illustrated". Signature 11 (1950): 4-18.

“Pleasing Decay”. Architectural Review 102 (septembre 1947): 85-94.

“Seaton Delaval". Orion: A Miscellany 1 (1945): 43-47.

“Towers in the Fens". Architectural Review (novembre 1940): 131-134

Table des illustrations:

Illustration 1 : Renishaw, the North Front (1942-5). Huile sur toile, 43,2x73,7cm. Sir Reresby Sitwell. Illustration 2 : View of Knole (1942-43). Huile sur toile, 53,3x76,2cm. Collection privée.

Illustration 3 : Coventry Cathedral, 15 November 1940 (1940). Huile sur toile, 76,2x63,5cm. Manchester City Art Gallery.

Illustration 4 : House of Commons 1941: Aye Chamber (1941). Huile sur toile. 75,7x63,5cm. National Gallery of Canada, Ottawa.

Illustration 5 : Somerset Place, Bath (1942) Aquarelle, 48,9x76,2cm. Tate Gallery.

Illustration 6 : Seaton Delaval (1941). Huile sur toile, $63,5 \times 76,2 \mathrm{~cm}$. Tate Gallery.

\section{NOTES}

1. Voir en particulier : Richard Ingrams, Piper's Places, London: Chatto \& Windus, 1983 ; David Fraser Jenkins, John Piper: The Forties, London: Philip Wilson Publishers \& Imperial War Museum, 2000 ; David Fraser Jenkins \& Frances Spalding, John Piper in the 1930s. Abstraction on the Beach, London: Merrell, 2003 ; Orde Levinson, "Quality and Experiment": the prints of John Piper. A catalogue raisonné 1923-91, London: Lund Humphries, 1996.

2. Axis 7 (automne 1936): 6 et 9. Myfanwy Evans publie Axis 1 de 1935 à 1937, organe des artistes abstraits britanniques (tels que Ben Nicholson et Barbara Hepworth) et critiques d'art qui veulent définir ce courant.

3. Voir notamment les articles de Roger Fry "An Essay on Aesthetics" (1909) et "Retrospect" (1920) dans Vision and Design (1929).

4. Ces guides sont illustrés par les photographies de John Piper, qui photographie systématiquement statues, églises ou inscriptions depuis 1935.

5. A Dialogue Upon the Gardens of the Right Honourable Lord Viscount Cobham at Stow in Buckinghamshire (1757), Three Essays on Picturesque Beauty (1792).

6. An Essay on the Picturesque (1794). 
7. John Piper's Stowe, Westerham: Hurtwood Press \& the Tate Gallery, 1983, 7 (« Though I was not at school there, Stowe has been a great education to me »).

8. Levinson, op. cit., 27. Lors de sa première visite à Renishaw Hall, John Piper rencontre Evelyn Waugh qui lui propose d'illustrer Brideshead Revisited avec des paysages imaginaires mais il refuse. Piper est probablement le modèle du personnage Charles Ryder.

9. En mars 1942, la RAF bombarde Lubeck. Hitler riposte en avril 1942 par une série d'attaques aériennes sur de petites villes historiques : Exeter, Bath, Norwich, York, Canterbury.

10. "Picturesque Travel Illustrated", Signature 11 (1950): 6.

11. Voir l'éditorial de l'Architectural Review 90 (juillet 1941): 1-2.

12. Ibid., 6.

13. Voir aussi l'éditorial du numéro spécial "The End of Reconstruction" de l'Architectural Review 92 (juillet 1942): 3-4.

14. L. Campbell, Coventry Cathedral, Art and Architecture in Post-War Britain, Oxford: Clarendon Press, 1996.

15. Voir notamment les articles de John Piper, "John Sell Cotman", Architectural Review 92 (juillet 1942): 10 ; "Letter from Norwich" (janvier 1944) in Piper, John, Buildings and Prospects, London: The Architectural Press, 1948, 117 et 122 ; "Pleasing Decay", Architectural Review 102 (septembre 1947): 75 et 88.

16. “Seaton Delaval", Orion: A Miscellany 1 (1945): 43.

17. Voir "Pleasing Decay", op. cit.

18. "Abstraction on the Beach", $X X^{e}$ siècle, 1er juillet 1938.

19. Voir Richard Ingrams, Piper's Places, chapitre 2 ("Abstraction Becomes a Place, 1936-8"), 12-19.

20. Arnold Palmer (ed.), Recording Britain, Oxford: OUP \& The Pilgrim Trust, volume 1 (1946), v.

21. Ibid., v («the outward aspect of Britain was changing all too quickly before the War at the sinister hands of improvers and despoilers »).

22. A. Bermingham, "System, Order and Abstraction: The Politics of English Landscape drawing around 1795", Landscape and Power, W.J.T. Mitchell (ed.), Chicago: Chicago UP, (1994) 2002, 77.

23. Sur la volonté politique d'occulter les pertes humaines et de mettre l'accent sur la reconstruction et la planification urbaine dès 1941, voir Campbell, Louise, op. cit., 9-10.

24. "The Architecture of Destruction", Architectural Review (juillet 1941): 25. Voir aussi «Pleasing Decay ", op. cit.

25. J.M. Richards, The Bombed Buildings of Britain: a Record of Architectural Casualties, London: The Architectural Press, 1942, 2.

26. "John Sell Cotman", op. cit., 10 (" he invented a formal language in which every particular has its due »).

27. J. Piper, British Romantic Artists, London: William Collins, 1942, 29.

28. Piper cite Ruskin dans "Pleasing Decay", op. cit., 93. Voir aussi "Decrepit Glory: a tour of Hafod" in J. Piper, Buildings and Prospects, op. cit.

29. "Towers in the Fens", Architectural Review (novembre 1940): 131.

30. "Pleasing Decay", op. cit. 85, 93, 94.

31. "Letter from Norwich", op. cit., 123 (" the war has arrested the rot of "development" »).

32. David D. Mellor (ed.), A Paradise Lost: The Neo-Romantic Imagination in Britain, 1935-1955, London: Lund Humphries \& The Barbican Art Gallery, 1987 (chap. "John Piper: Mapping out British culture"), 34-39.

33. J. Piper, "Art or Anti-Art? " in J. Osborne, John Piper and the Stained Glass, Stroud: Sutton, 1997, 158-160.

34. Voir "Towers in the Fens", op. cit., 131, et "The Architecture of Destruction", op. cit., 25 ("Crackle of glass underfoot"). 
35. C. Hussey, "The Twilight of the Great House", Country Life (January 1945): 153. Citation du catalogue d'exposition des toiles que John Piper a peintes (entre autres) pour illustrer l'autobiographie de Sir Osbert Sitwell.

36. F. McCormick, Sir John Vanbrugh. The Playwright as Architect, University Park: Pennsylvania State UP, 1991. Voir aussi "Seaton Delaval", op. cit., 47.

37. Voir en particulier : John Martin Robinson, The Country House at War, London: Bodley Head, 1989 ; Roy Strong, Marcus Binney \& John Harris (eds.), The Destruction of the Country House, 1875-1975, London: Thames \& Hudson, 1974 ; Merlin Waterson (ed.), The Country House Remembered: Recollections of Life Between the Wars, London: Routledge \& Kegan Paul, 1985.

INDEX

oeuvrecitee Recording Britain 